\title{
Mechanical and histological properties of an electrospun scaffold with a modified surface by plasma polymerization implanted in an in vivo model
} \author{
DIEGO R. ESQUILIANO-RENDÓN ${ }^{3}$ \\ ${ }^{1}$ Research Vice-Rectory, Faculty of Engineering, University La Salle México, 06140, México \\ 2 Department of Physics, Metropolitan Autonomous University, 09340, México \\ 3 Department of Tissue Engineering, Child Hospital of México Federico Gómez, 06720, México
}

María G. FLORES-SÁNCHEZ ${ }^{1 *}$; Roberto OLAYO²; J. MORALES-CORONA²; ATLÁNTIDA M. RAYA-RIVERA³;

Key words: Hydroxyapatite, Polylactic acid, pyrrole, Electrospinning, Surface activation

\begin{abstract}
This article presents the construction of scaffolds composed of polylactic acid (PLA) with different concentrations of hydroxyapatite (HA) by electrospinning, which were superficially modified with polypyrrole (PPy/I) by plasma polymerization. A preliminary study was conducted of the biological and mechanical behavior of the scaffolds when they were implanted in the back of rabbits for 30 days; bone cells differentiated from mesenchymal stem cells (MSCs) were used. The bone cell and scaffold structures were characterized by histological, immunohistochemical, and mechanical stress tests. Hematoxylin-eosin staining showed good tissue conformation. The immunohistochemical tests highlighted the presence of the main bone tissue proteins, such as collagen, osteocalcin, and osteopontin. The PLA/HA scaffolds were observed to exhibit cell adhesion and proliferation properties; however, the response was much better in the scaffolds that had a higher concentration of HA and that were coated with PPy/I. The results of the mechanical tests of the scaffolds indicated that the plasma treatment improved the adhesion and cell proliferation properties and contributed to the mechanical support, allowing the formation of neotissues with good viability of cell growth.
\end{abstract}

\section{Introduction}

In bone tissue engineering, scaffolds serve as tissue formation matrices, and it is desirable that they meet basic characteristics that allow cell adhesion, differentiation and proliferation, mechanical integrity, osteoconductivity and biocompatibility, vascularization, and nutrients and bioactive factors (de Witte et al., 2018; Hosseinpour et al., 2017; Motamedian et al., 2015; Venkatesan et al., 2017). Ceramicpolymeric compounds have given good results as scaffolds in in vitro and in vivo studies (Asghari et al., 2017; Naahidi et al., 2017; Qi et al., 2016; Tajbakhsh and Hajiali, 2017). The combination of hydroxyapatite (HA) and polylactic acid (PLA), in particular, has shown the ability to promote new bone generation because they form porous scaffolds that exhibit the superimposed behavior of ceramics in a polymeric

\footnotetext{
*Address correspondence to: María G. Flores-Sánchez, guadalupe.flores@lasalle.mx

Received: 17 April 2021; Accepted: 07 June 2021
}

matrix, which has good bioactivity, osteoconductivity, and osteoinductivity (Lei et al., 2017; Mao et al., 2018; Mondal et al., 2016; Quinlan et al., 2015; Zhang et al., 2018). In addition, both materials are biocompatible, and the polymer helps morphogenetic proteins to induce bone tissue formation (Mao et al., 2018; Narayanan et al., 2016; Samadikuchaksaraei et al., 2016; Sha et al., 2016). In this study, polypyrrole (PPy/I) was considered as a cell adhesion factor, since it is a conjugated polymer with good conductivity. Particularly in the field of biomaterials, the plasma polymerization technique has been used to superficially modify biomaterials. The technique uses a reactor with specific pressure, frequency, temperature, time, and power conditions, in which the monomer is introduced as a vapor that ionizes in the reaction and polymerizes through complex mechanisms (Cruz et al., 2010; Friedrich, 2011). Polymers produced by plasma polymerization are not chemically regular like conventional polymers but are rich in functional groups, which can serve as chemical anchors or facilitate the interaction with other materials, including biological media. Moreover, these groups can interact with various cells, allowing their anchorage, differentiation, and 
proliferation and even promoting cell regeneration (AlvarezMejia et al., 2015; Olayo et al., 2008).

Another important aspect in this study was the incorporation of bioactive cells into the three-dimensional porous scaffold from mesenchymal stem cells (MSCs). MSCs are present in many human tissues and can be derived directly from bone marrow and isolated, grown, and proliferated in the laboratory. These cells are capable of giving rise to various tissues, such as bone, muscles, and other tissues of mesenchymal origin. In addition, MSCs do not appear to be rejected by the immune system (Chuenjitkuntaworn and Pavasant 2015; Hoda et al., 2016; Elkhenany et al., 2014; Yousefi et al., 2016).

\section{Main Text}

This study presents the evaluation of the biological and mechanical behavior of biomaterials composed of $\mathrm{HA}$ and PLA superficially modified with PPy/I after being implanted in vivo in the back of rabbits for 30 days. Good osteoinductive activity of the neotissues obtained was observed because of PPy/I and the amount of HA they possessed, which was reflected in the presence of proteins such as collagen, osteocalcin, and osteopontin. Furthermore, it was observed that when the materials had less HA and PLA, their Young's modulus was higher owing to the low rigidity of the ceramic. However, better cell viability was shown in materials that had more HA, so it is important to take care of the HA-to-PLA ratio to obtain a material that has good mechanical properties and that promotes osteogenesis. Therefore, scaffolds composed of PLA, HA, and PPy/I are considered a promising alternative for the construction of new tissues with osteogenic lineages. It is important to highlight that the function of HA and PLA is to work as temporal scaffold that gives structural support to the cells, while PPy improves the adhesion and cell proliferation properties. Once the material is implanted in vivo, HA is integrated by bone cells, while PLA is degraded down into its non-toxic elements.

\section{Materials and Methods}

Preparation of the scaffolds of PLA/HA and PLA/HA/PPy/I Precursor polymer solution was prepared by dissolving $1.8 \mathrm{~g}$ of PLA (molecular weight $65,112 \mathrm{~g} / \mathrm{mol}$ ) in a chloroform/ ethanol (90/10) mixture. The solution was gently stirred for $5 \mathrm{~h}$ at room temperature, after which HA was dispersed to obtain concentrations of $18.1 \%$ and $35.7 \% \mathrm{w} / \mathrm{w}$ (HA/PLA). The scaffolds obtained were removed from the collector and dried at $42^{\circ} \mathrm{C}$ under vacuum conditions for 4 days to remove excess solvent. Electrospinning technique was used at room temperature to manufacture porous PLA/HA matrices (control group). These matrices were spun at HA concentrations of $18.1 \%$ (sample group M1) and $35.7 \%$ (sample group M2). The resulting porous materials were duplicated and coated with PPy doped with iodine by plasma polymerization (PLA/HA/PPy/I) at the same HA concentrations (sample groups M3 and M4).

\section{Plasma polymerization}

Plasma polymerization technique was used to synthesize $\mathrm{PPy} / \mathrm{I}$ films and to modify the physicochemical properties of the surfaces of the PLA/HA samples (M3 and M4). The PLA/HA scaffolds were placed in the middle of the plasma polymerization reactor, in which the electrode spacing was approximately $10 \mathrm{~cm}$. Pyrrole was introduced, and iodine (I) was used as a dopant; the experiment lasted $30 \mathrm{~min}$. The polymerization conditions were as follows: $13.5 \mathrm{MHz}, 20 \mathrm{~W}$, and $1.2 \times 10^{-2}$ Torr; the initial temperature of the monomers was approximately $25^{\circ} \mathrm{C}$. The purpose of polymerize $\mathrm{PPy} / \mathrm{I}$ by using plasma technique is to activate the scaffold surfaces.

\section{Mechanical stress tests of the scaffolds of PLA/HA and PLA/HA/PPy/I}

Mechanical properties of the fabricated scaffolds were analysed with a single-axis machine. Rectangular samples with an average dimension of $5 \times 20 \mathrm{~mm}$ and $0.2 \mathrm{~mm}$ thick were measured. Each scaffold was isolated, prepared, and assembled for tension testing, under ambient conditions of temperature and humidity, at a speed of $0.5 \mathrm{~mm} / \mathrm{min}$ until its fracture. The tensile tests of the composite materials were carried out in accordance with ASTM D 638, ASTM D 3039, and ASTM C 297.

Isolation, culture, and characterization of bone marrow stem cells (MSCs)

MSCs were obtained by aspirating $3 \mathrm{~mL}$ of $2 \mathrm{~kg}$ of male New Zealand rabbit bone marrow. The animals were relaxed with $20 \mathrm{mg} / \mathrm{kg}$ of ketamine and $5 \mathrm{mg} / \mathrm{kg}$ of acepromazine; then, they were anesthetized with sodium pentobarbital $(40 \mathrm{mg} / \mathrm{kg})$. Each sample underwent a density gradient test with NycoPrep $(1077 \mathrm{~g} / \mathrm{mL})$ and was centrifuged at $600 \mathrm{rpm}$ $\times 20 \mathrm{~min}$ to separate the different cell types. Subsequently, the fraction corresponding to the MSCs was recovered, and the cell buttons were seeded in Petri dishes with an $\alpha$-MEM medium supplemented with $10 \%$ SFB and incubated under standard culture conditions: $37^{\circ} \mathrm{C}, 5 \% \mathrm{CO}_{2}$ atmosphere, and saturated humidity. The characterization of the MSCs was performed with alkaline phosphatase staining and by flow cytometry using the antibodies CD45, CD34, HLA-DR, CD90, and CD73.

Differentiation of MSCs into an osteogenic lineage and their seeding in PLA/HA and PLA/HA/PPy/I templates

Differentiation was performed under standard culture conditions for $24 \mathrm{~h}$ in an osteogenic differentiation medium. The cell medium was then exchanged for a fresh medium. The cells obtained were detached from the Petri dishes by means of a trypsin-EDTA solution, and this was inactivated with serum. The cells were subsequently centrifuged, and the button was resuspended in an a-MEM culture medium supplemented with $15 \%$ inactivated FBS and a $1 \%$ antibiotic-antifungal solution. The cells were seeded in each of the PLA/HA and PLA/HA/PPy/I scaffolds of $5 \times 15 \mathrm{~mm}$ at a concentration of $2.5 \times 10^{6}$ cells per box. The scaffolds and cells were incubated for $1 \mathrm{hr}$ to stimulate the adherence of the cells. Subsequently, they were resuspended with a DMEM medium and kept in culture for 7 days (changing the medium every 3 days for a fresh medium), and on the seventh day we proceeded with implantation in vivo (Hayami et al., 2003). 
Cell viability of the scaffolds before implantation

Cell viability of the neotissues was evaluated 7 days after cell seeding by the 3-[4,5-Dimethylthiazol-2-yl]-2,5-diphenyltetrazolium bromide (MTT) colorimetric method. The neotissues were incubated at $37^{\circ} \mathrm{C}$ for $3 \mathrm{~h}$ in the dark in $1 \mathrm{~mL}$ of DMEM/F12 medium without serum with $200 \mu \mathrm{L}$ of PBS-MTT $(5 \mathrm{mg} / \mathrm{mL})$. After some time, the medium was removed; the templates were washed with $\mathrm{PBS}$, and then $400 \mu \mathrm{L}$ of isopropanol-HCl (0.04\%) was added. The supernatants obtained were read at an optical density (OD) of $595 \mathrm{~nm}$ in a TECAN GENios reader.

Implantation of the scaffolds and mechanical tests

Autologous implantation was performed in the rabbits from which bone marrow cells were extracted. The scaffolds and cells were placed in a vicryl mesh and implanted subcutaneously on the back of the animals, fixed at the hip with non-absorbable sutures. Rectangular samples of the tissues with an average dimension of $10 \times 30 \mathrm{~mm}$ were removed 30 days after implantation; they were extracted from the animal on the same day. Each scaffold was tested in a single-axis tension machine, under ambient conditions of temperature and humidity, at a speed of $0.5 \mathrm{~mm} / \mathrm{min}$ until its fracture. The tensile tests of the composite materials were carried out in accordance with ASTM D 638, ASTM D 3039, and ASTM C 297.

\section{Histological and immunohistochemical analysis of the removed tissues}

The tissues were subjected to the process of fixation with $4 \%$ formalin, followed by embedding in paraffin. Tissue sections of $5 \mu \mathrm{m}$ were handled and stained with hematoxylin and eosin. Other tissue sections were used for immunohistochemistry. Osteoblastic cells were identified with anti-rabbit polyclonal reagents (type I collagen and osteocalcin) at a dilution of 1:50 kept for $24 \mathrm{~h}$ at $4^{\circ} \mathrm{C}$. The preparations were counter-stained with hematoxylin.

\section{Results}

\section{Infrared spectrophotometry}

The scaffolds were superficially modified by plasma polymerization, resulting in the deposition of a thin layer of material with various functional groups, such as amines $(\mathrm{NH}$, $\mathrm{NH}_{2}$ ), nitriles, and aliphatic groups (Alvarez-Mejia et al., 2015). Fig. 1 shows the spectrum of PLA with its characteristic vibrations around $1,000-2,000 \mathrm{~cm}^{-1}$. Centered at $1,046 \mathrm{~cm}^{-1}$ is the vibration of C-O. At $1,750 \mathrm{~cm}^{-1}$, the stretching of the $\mathrm{C}=\mathrm{O}$ and the $\mathrm{C}-\mathrm{O}$ bond of the ester group is observed, and around $2,950 \mathrm{~cm}^{-1}$, the characteristic vibration of aliphatic C-H carbons, are seen. The IR spectra with HA and PPy/I show a region between 3,100 and $3,450 \mathrm{~cm}^{-1}$, which can be attributed to the presence of the $\mathrm{OH}$ and $\mathrm{NH}$. The spectra with $\mathrm{PPy} / \mathrm{I}$ show a vibration at $1,600 \mathrm{~cm}^{-1}$, which corresponds to the $\mathrm{C}=\mathrm{C}$ and $\mathrm{NH}$ groups of pyrrole ring.

Tension tests of the PLA-PHA and PLA-HA-PPy/I scaffolds Fig. 2 shows the results of the stress tests on the different electrospun scaffolds, in which the maximum stress, strain, and Young's modulus were measured.

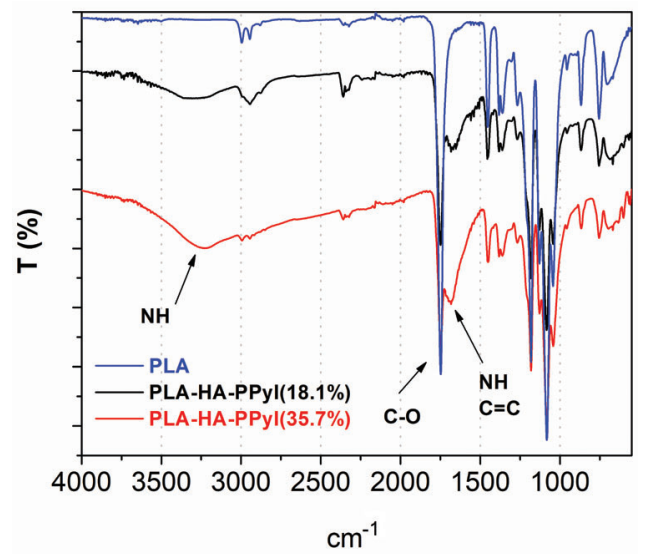

FIGURE 1. Spectra with PPy/I show vibrations at $1,600 \mathrm{~cm}^{-1}$ and from 3,000 to $3,500 \mathrm{~cm}^{-1}$, which correspond to the vibrations of the $\mathrm{OH}$ groups of $\mathrm{HA}$ and the vibrations of the $\mathrm{NH}$ groups of PPy/I, respectively.

Fig. 3 shows coated film obtained with the plasma polymerization technique. Compared with those of PLA (Fig. 3A), the samples of PLA-PPy/I have lower values for all the three characteristics, since the samples with PPy/I contain fibers that make the structure sensitive to rupture (Fig. 3B, red circles). Regarding the PLA-HA (18.1\%) and PLA-HA (18.1\%)-PPy/I scaffolds, the former supports more stress and has a higher deformation and Young's modulus than the scaffolds that have PPy/I, which tend to break faster. This can be attributed to the cracked fibers (red circles) that occur in these scaffolds, since the thin layer of PPy/I that covers the surface of the fibers can cause the fibers to stiffen, so not allowing them to stretch when deformed by the applied stress (Fig. 3C). On the other hand, the PLA-HA (35.7\%) and PLA-HA (35.7\%)-PPy/I scaffolds support the least load, since both have many clusters thanks to HA. These clusters withstand rupture, but once the breaking point is reached, they break completely. The scaffold with PPy/I shows an almost double deformation, is more fragile, and breaks until the total rupture (Fig. 3D).

\section{Mechanical analysis of the removed neotissues}

Fig. 4 is made up of Columns A-B and Rows I-IV. Columns A-B show the results of the stress tests of the neotissues. When the tension load was applied, the removed neotissues were stretched, showing local effort in various areas and maximum effort, followed by a decrease in effort and then recovery. The neotissues were found to be well integrated and vascularized; however, there was a noticeable difference in the deformation of the neotissues with the least amount of HA, since the ceramic clusters produced hollow areas where the tension force acted, tending to disrupt the tissue abruptly and spreading to the rest of the tissue until it broke completely.

Neotissue M1 obtained the highest stress (1.6 MPa), and the elasticity limit reached 95\%. Neotissue M2 (with PPy/I) presented a stress of $1.2 \mathrm{MPa}$ and an elasticity limit of $50 \%$, almost half of that of M1. This difference can be attributed to the presence of HA and PPy/I: The hollow sections in these materials cause the tissue to break faster and more abruptly. Young's modulus of M1 was slightly higher than 
(A)

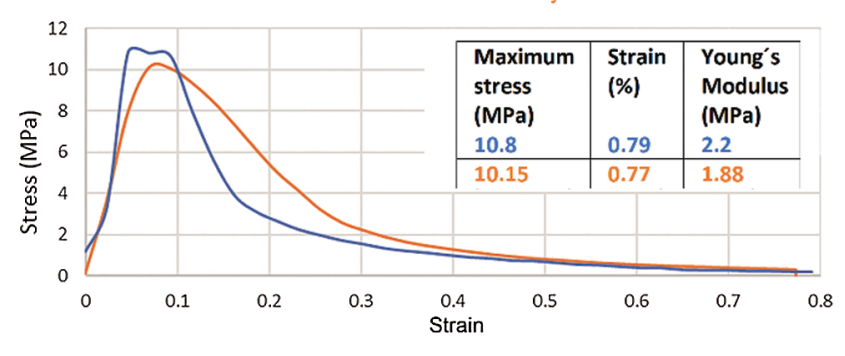

(B) 8

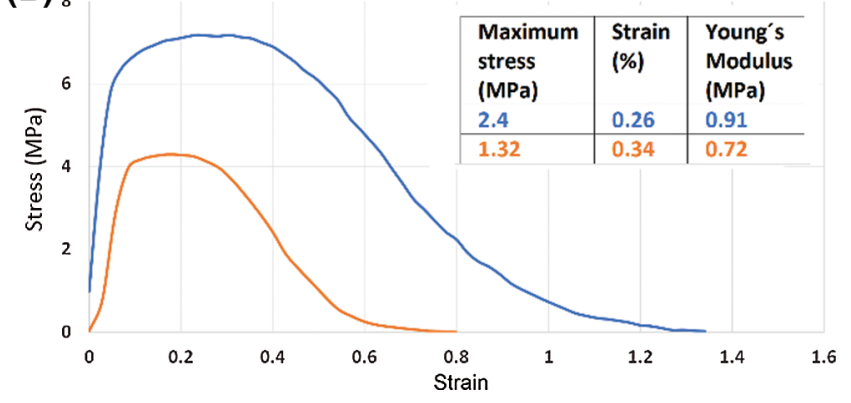

(C)

PLA-HA(35.7\%) and PLA-HA (35.7\%)-PPy/l

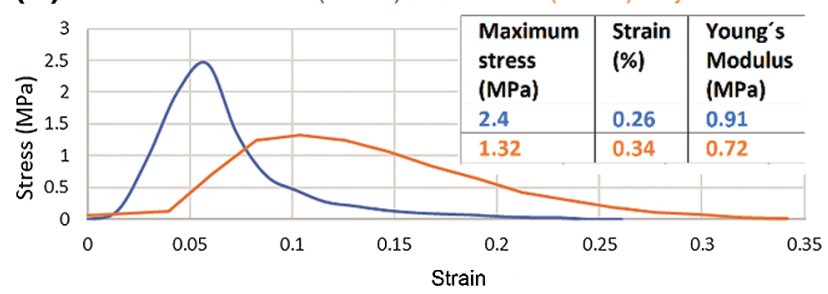

FIGURE 2. Stress-strain graphs of PLA/HA (18.1\%), PLA/HA (18.1\%)/PPy/I, PLA/HA (35.7\%), PLA/HA (35.7\%)/PPy/I, showing elastic deformation and local stress until reaching the maximum stress. HA and PPy/I contribute to a decrease in load and deformation that the materials can support until reaching rupture.

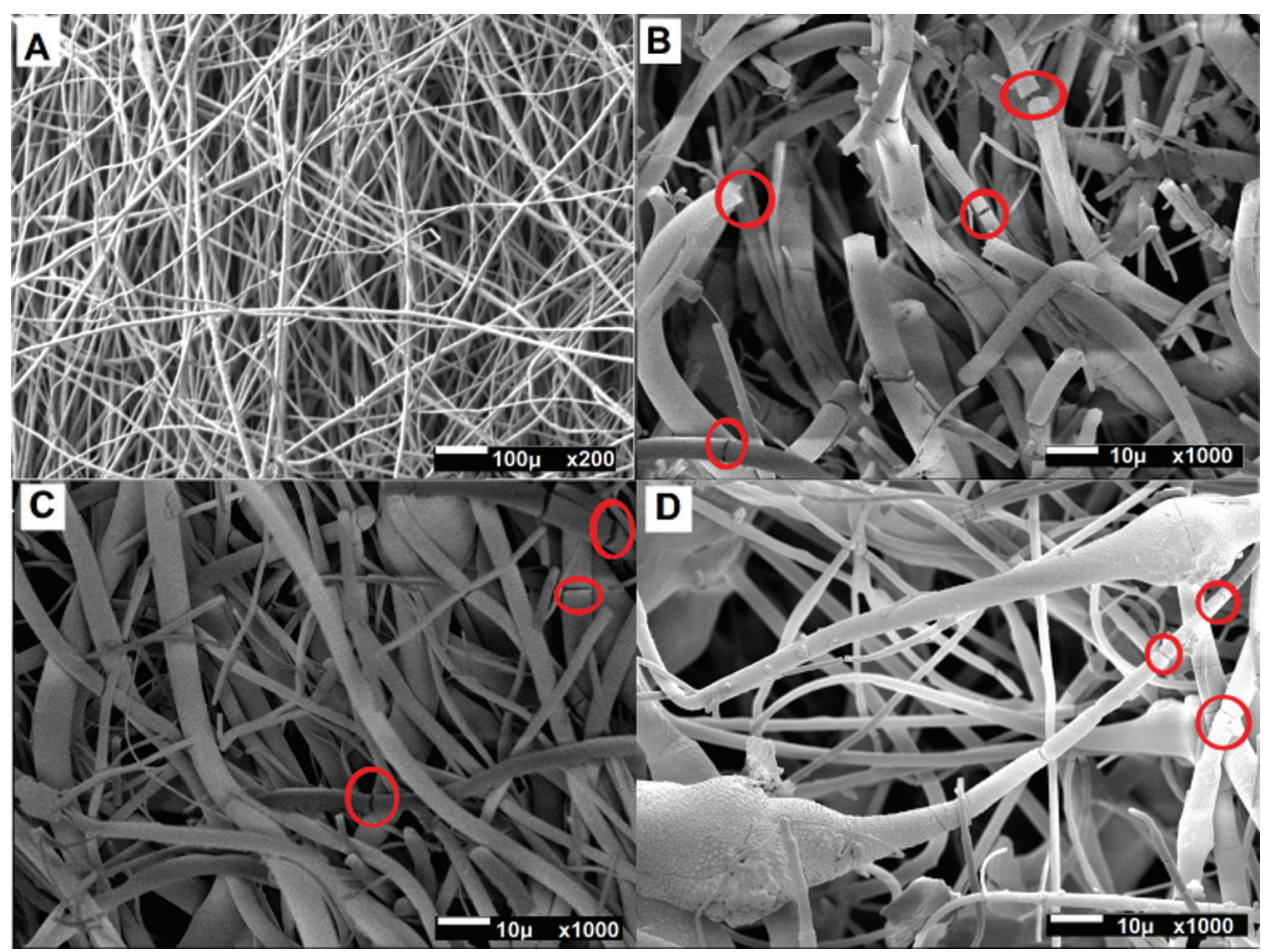

FIGURE 3. SEM of coating films. (A) PLA/PPy/I presents smooth fibers and a homogeneous morphology and reaches a higher deformation. (B) PLA/HA (18.1\%)/PPy/I shows fibers, which make them more prone to rupture. (C) PLA/HA (18.1\%)/PPy/I; the samples with $\mathrm{PPy} / \mathrm{I}$ present cracked fibers (red circles). (D) PLA/ HA (35.7\%)/PPy/I; the samples with more HA present clusters of this ceramic, which cause abrupt rupture in some areas (red circles). that of $\mathrm{M} 2$, with values of 0.84 and $0.8 \mathrm{MPa}$, respectively (Figs. 4A-I and 4B-I, Figs. 4A-II and 4B-II).

Neotissue M3, without PPy/I but with more HA, showed a maximum stress of $0.5 \mathrm{MPa}$ and a brittle stress-strain profile with $60 \%$ strain. For M4 maximum stress was of $0.4 \mathrm{MPa}$ and the presence of PPy/I maintained the strength of the scaffolds but considerably reduced the deformation to $30 \%$. Young's modulus was also slightly reduced compared with that of $\mathrm{M} 3$, with values of 0.47 and $0.307 \mathrm{MPa}$, respectively (Figs. 4A-III and 4B-III, Figs. 4A-IV and 4B-IV).

The mechanical properties of neotissues are controlled by several microstructural factors, such as the properties of the matrix, the distribution of the areas covered with tissue, the bond strength, and the methods of processing and obtaining the matrices. The strength of the interfaces of PLA-HA and PLA-HA-PPy/I greatly affects the efficiency of the charge transfer to the matrix.

By adding PPy/I, we can produce scaffolds with a lower Young's modulus; moreover, the addition of HA further increases the brittleness of the neotissue. In contrast, scaffolds with lower HA are more flexible and present a higher Young's modulus. The concentration of HA and the presence of PPy/I resulted in significant differences in the interactions when the neotissues were stressed, at least for this type of in vivo culture 


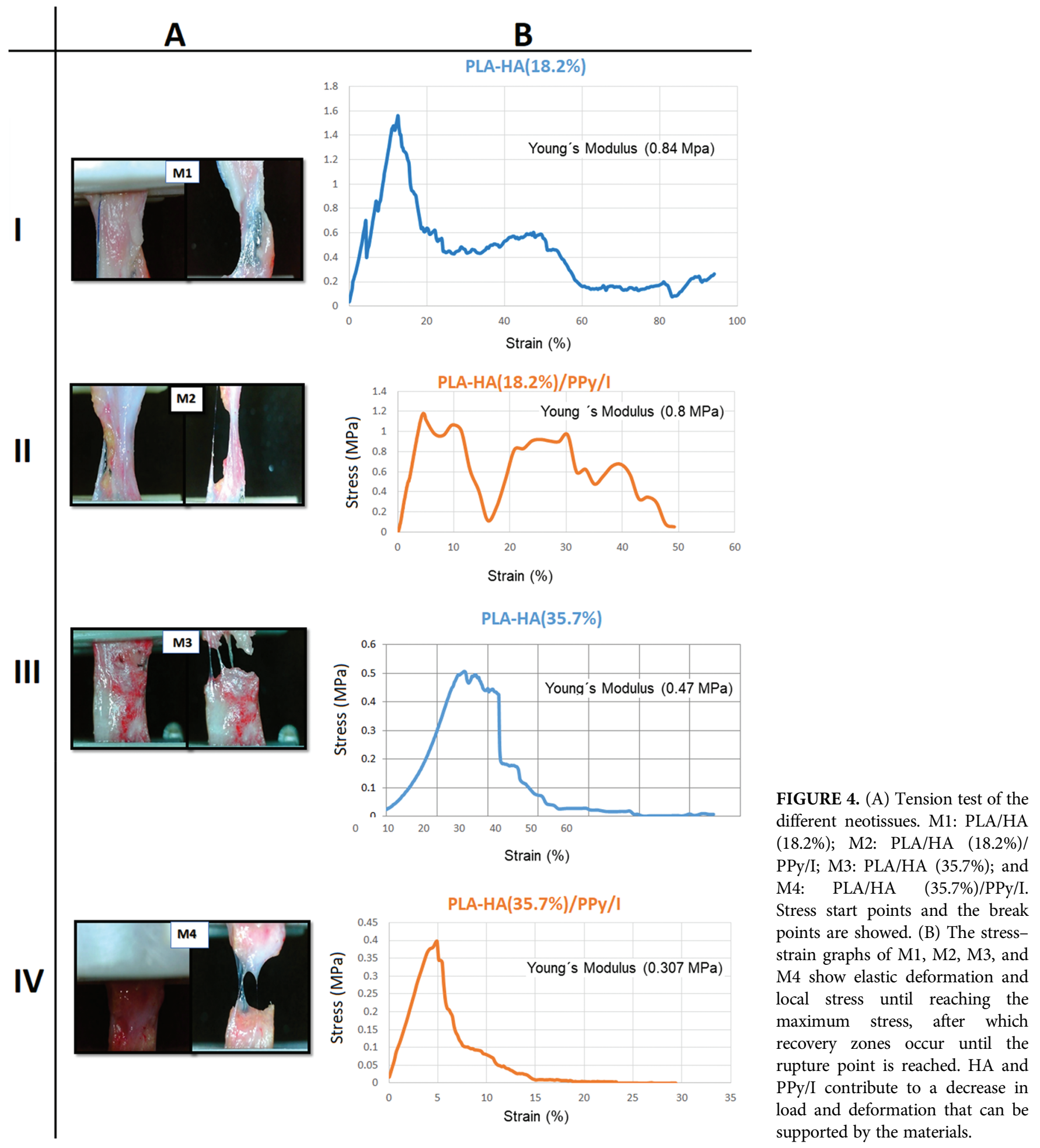

of 30 days. In these tests, the elastic modulus of the scaffolds decreased with increasing mixing ratios, which enhanced the contribution of the different materials in the general conformation.

One more test for analyzing scaffolds and helping bone tissue engineering is the scaffold's ability to support bone-like extracellular matrix deposition. To this end, collagen, osteocalcin, osteopontin, and matrix staining with hematoxylin $(\mathrm{H})$ and eosin (E) were used to study the production of an extracellular matrix during an in vivo culture of 30 days. In Fig. 5, it can be seen that in M1, the H-E staining shows areas with cell nuclei. Immunohistochemistry shows abundant collagen expression (brown color), which shows the presence of tissue differentiating into a bone lineage. Osteocalcin and osteopontin are also expressed in the brown areas; however, less formed areas and spaces between them can also be seen, with surrounding tissue of another type.

Something similar occurs with sample M2, which contains PPy/I. For this sample, a main difference in H-E staining can be observed since the sample greatly improved compared with M1: There are fewer empty areas, and the tissue is even more shaped, preserving the presence of collagen, osteocalcin, and osteopontin. 


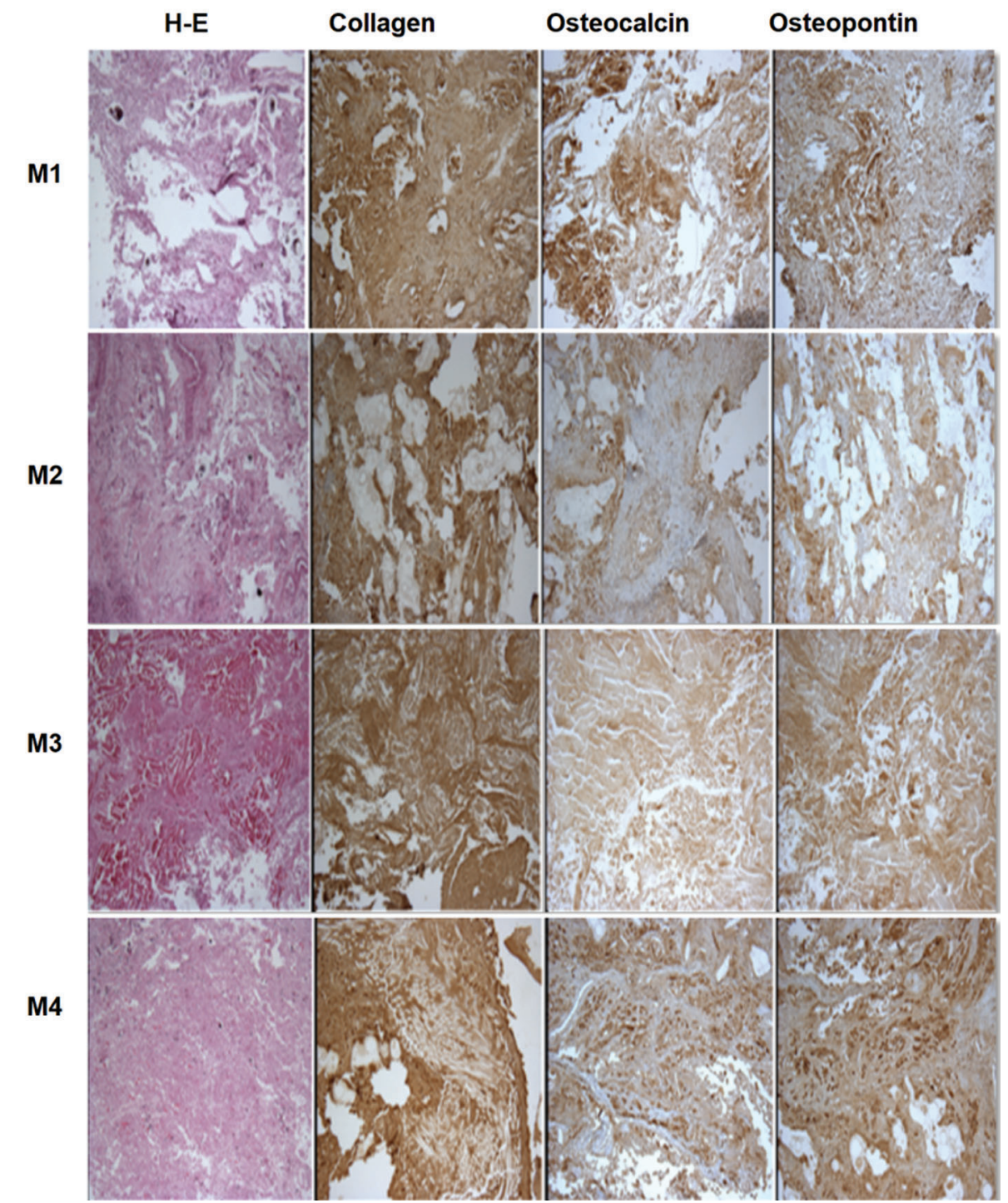

FIGURE 5. Histology and immunohistochemistry of the new tissues. The H-E, collagen, osteocalcin, and osteopontin tests confirmed that the scaffolds with the highest cell proliferation and tendency towards an osteogenic lineage are those with the highest HA content and covered with PPy/I, namely M3 and M4.

The production of collagen by bone cells in the scaffolds was higher for the M3 and M4 scaffolds than for the M1 and M2 scaffolds, although empty areas can be observed. This is due to the concentration of bone cells in certain parts, since tissue of another type was found in the surrounding area or part of the tissue was simply lost during histology, but in general, the cells were found to be distributed on the surface of all scaffolds. The histology of M3 and M4 confirmed the good cell viability shown by the MTT test prior to implantation (Flores-Sánchez et al., 2021).

\section{Cellular viability of the removed neotissues}

In general, cells were viable in all the scaffolds during the 7-day in vitro culture period. The M3 PLA/HA (35.7\%) and M4 PLA/ HA (35.7\%)/PPy/I samples had better cellular viability, but there was a difference between the samples that had PPy/I and those that lacked it. It should be noted that the balance of cartilage formation and vascular invasion is essential since cell proliferation plays a crucial role in early osteogenesis, in which characteristic proteins of the bone lineage, such as type I collagen, are expressed and in which osteocalcin and osteopontin participate in the formation of the extracellular matrix. The cell viability test of the removed neotissues showed their ability to mimic the natural three-dimensional environment of the extracellular matrix in vivo, which, while providing structural support with a high surface-to-volume ratio, builds excellent structures for engineering applications of tissues (Hayami et al., 2003).

\section{Discussion}

Polymeric matrices with higher values of Young's modulus and yield strength were obtained in M1 PLA/HA (18.1\%) and M2 PLA/HA (18.1\%)/PPy/I than in M3 PLA/HA (35.7\%) and M4 PLA/HA (35.7\%)/PPy/I. These differences are attributed to the amount of HA present and the surface modification induced by PPy/I. In M3 and M4, the best cell viabilities were found, as shown by histology and the MTT assay. In these samples, the greatest deposition of collagen was observed, and there was the labeling of osteocalcin and osteopontin, as well as the H-E staining of cell nuclei of an osteogenic lineage. 
In general, the contribution of $\mathrm{PPy} / \mathrm{I}$ in the removed neotissues was not significant for the mechanical properties of the neotissues that were found to be made up of hard and soft segments. The HA aggregates and the PPy/I cover in the neotissues probably created regions of large crosssections of fibers and clusters, which could have caused a greater amount of material in relation to the empty space within the sample. This in turn creates a more solid scaffold but with a reduced capacity to undergo tension, at least for this evaluation period of only 30 days after implantation. However, morphologically, there are more closed tissues capable of stimulating adhesion and cell proliferation, which shows that the cells cover areas on the surface of the scaffold, but it takes more time to form a more resistant tissue. Therefore, it is necessary that HA is present in the scaffolds and that the surface of the scaffolds is superficially modified by PPy/I so that the neotissues in which the formation of bone matrix is supported are enhanced. It was also observed that these neotissues show vascularization, absence of necrosis, and good conformation. Increasing the duration of the experiment and implanting it in bone will allow to establish osseointegration and a better evaluation of the biomaterials. In summary, neotissues were obtained with the expression of proteins characteristic of bone tissue, with good vascularization and cell proliferation, making these scaffolds a viable alternative for the possible management of defects in long bones. Finally, Infrared spectrophotometry showed that the scaffolds contain the components corresponding to the functional groups of HA and PLA; that is, they have sufficient chemical diversity to enrich the surface of the matrices, resulting in a better shaped structure and promoting links that provide mechanical stability to the scaffold.

\section{Conclusion}

Polymeric matrices with higher values of Young's modulus and yield strength were obtained in M1 PLA/HA (18.1\%) and M2 PLA/HA (18.1\%)/PPy/I than in M3 PLA/HA (35.7\%) and M4 PLA/HA (35.7\%)/PPy/I, allowing better handling of samples when working on implants. These differences are attributed to the amount of HA present and the surface modification induced by PPy/I. In M3 and M4, the best cell viabilities were found, as shown by the histologies, in these samples, the greatest deposition of collagen was observed, and the labeling of osteocalcin and osteopontin was also present, as well as the H-E staining of cell nuclei of an osteogenic lineage. Therefore, the presence of HA in the scaffolds and the surface modification of the scaffolds by PPy/I allow the generation of neotissues in which the formation of bone matrix is supported are enhanced. It was also observed that these neotissues show vascularization, absence of necrosis, and good conformation.

Acknowledgement: We thank Dr. Patricia Ontiveros Nevares of the Morelos Children's Hospital for her contribution to the histological and immunohistochemical studies.

Availability of Data and Materials: The data that support the findings of this study are available on request from the corresponding author [María G. Flores-Sánchez].
Author Contribution: The authors confirm contribution to the paper as follows: study conception and design: MGF-S, RO, MAR-R; data collection: MGF-S, RO, JM, DRE-R; analysis and interpretation of results: MGF-S, RO, MAR-R. MGF-S. All authors reviewed the results and approved the final version of the manuscript.

Ethics Approval: The ethical standards of existing international health research, established for handling animals, were followed. All animal experiments comply with the National Institutes of Health guide for the care and use of laboratory animals (NIH Nos. 8023, 1978).

Funding Statement: This work was supported by Universidad La Salle México and the Faculty of Engineering, Grant No. EC388-IMC-13/18; CONACYT, Grant No. CB16RF_287927.

Conflicts of Interest: The authors declare that they have no conflicts of interest to report regarding the present study.

\section{References}

Alvarez-Mejia L, Morales J, Cruz GJ, Olayo MG, Olayo R et al. (2015). Functional recovery in spinal cord injured rats using polypyrrole/iodine implants and treadmill training. Journal of Materials Science: Materials in Medicine 26: S2. DOI 10.1007/s10856-015-5541-0.

Asghari F, Samiei M, Adibkia K, Akbarzadeh A, Davaran S (2017). Biodegradable and biocompatible polymers for tissue engineering application: A review. Artificial Cells, Nanomedicine, and Biotechnology 45: 185-192. DOI 10.3109/21691401.2016.1146731.

Chuenjitkuntaworn B, Pavasant P (2015). The efficacy of polycaprolactone/hydroxyapatite scaffold in combination with mesenchymal stem cells for bone tissue engineering. Journal of Biomedical Materials Research Part A 104: 264271. DOI 10.1002/jbm.a.35558.

Cruz GJ, Olayo M, López OG, Gómez LM, Morales-Corona Juan et al. (2010). Nanospherical particles of polypyrrole synthesized and doped by plasma. Polymer 51: 4314-4318. DOI 10.1016/j.polymer.2010.07.024.

de Witte TM, Fratila-Apachitei LE, Zadpoor AA, Peppas NA (2018). Bone tissue engineering via growth factor delivery: From scaffolds to complex matrices. Regenerative Biomaterials 5: 197-211. DOI 10.1093/rb/rby013.

Elkhenany H, Amelse L, Lafont A, Bourdo S, Caldwell M et al. (2015). Graphene supports in vitro proliferation and osteogenic differentiation of goat adult mesenchymal stem cells: Potential for bone tissue engineering. Journal of Applied Toxicology 35: 367-374. DOI 10.1002/jat.3024.

Flores-Sánchez MG, Islas-Arteaga NC, Raya-Rivera AM, EsquilianoRendon DR, Morales-Corona J et al. (2021). Olayo R Effect of a plasma sinthesized polypyrrole coverage on polylactic acid/ hydroxyapatite scaffolds for bone tissue engineering. Journal of Biomedical Materials Research Part A 109: 2199-2211. DOI 10.1002/jbm.a.37205.

Friedrich J (2011). Mechanisms of plasma polymerizationReviewed from a chemical point of view. Plasma Processes and Polymers 8: 783-802. DOI 10.1002/ppap.201100038.

Hayami T, Funaki H, Yaoeda K, Mitui K, Yamagiwa H et al. (2003). Expression of the cartilage derived anti-angiogenic factor chondromodulin-I decreases in the early stage of experimental osteoarthritis. Journal of Rheumatology 30: 2207-2217. 
Hoda E, Amelse L, Caldwell M, Abdelwahed R, Dhar M (2016). Impact of the source and serial passaging of goat mesenchymal stem cells on osteogenic differentiation potential: Implications for bone tissue engineering. Journal of animal science and biotechnology 7: 16. DOI 10.1186/s40104-016-0074-z.

Hosseinpour S, Ghazizadeh Ahsaie M, Rezai Rad M, Baghani MT, Motamedian SR et al. (2017). Application of selected scaffolds for bone tissue engineering: A systematic review. Application of selected scaffolds for bone tissue engineering: A systematic review. Oral and Maxillofacial Surgery 21: 109-129. DOI 10.1007/s10006-017-0608-3.

Lei Y, Xu Z, Ke Q, Yin W, Chen Y et al. (2017). Strontium hydroxyapatite/chitosan nanohybrid scaffolds with enhanced osteoinductivity for bone tissue engineering. Materials Science and Engineering: C 72: 134-142. DOI 10.1016/j. msec.2016.11.063.

Mao D, Qing L, Ningning B, Hongzhou D, Daikun L (2018). Porous stable poly(lactic acid)/ethyl cellulose/hydroxyapatite composite scaffolds prepared by a combined method for bone regeneration. Carbohydrate Polymers 180: 104-111. DOI 10.1016/j.carbpol.2017.10.031.

Mondal S, Umapada P, Apurba D (2016). Natural origin hydroxyapatite scaffold as potential bone tissue engineering substitute. Ceramics International 42: 18338-18346. DOI 10.1016/j.ceramint.2016.08.165.

Motamedian SR, Hosseinpour S, Ahsaie MG, Khojasteh A (2015). Smart scaffolds in bone tissue engineering: A systematic review of literature. World Journal of Stem Cells 7: 657-668. DOI 10.4252/wjsc.v7.i3.657.

Naahidi S, Mousa J, Megan L, Yujie W, Yongfang Y et al. (2017). Biocompatibility of hydrogel-based scaffolds for tissue engineering applications. Biotechnology Advances 35: 530544. DOI 10.1016/j.biotechadv.2017.05.006.

Narayanan G, Vernekar VN, Kuyinu EL, Laurencin CT (2016). Poly (lactic acid)-based biomaterials for orthopaedic regenerative engineering. Advanced Drug Delivery Reviews 107: 247-276. DOI 10.1016/j.addr.2016.04.015.

Olayo R, Ríos C, Salgado-Ceballos H, Cruz GJ, Morales J, Olayo MG, Alcaraz-Zubeldia M, Alvarez AL, Mondragon R, Morales A, Diaz-Ruiz A (2008). Tissue spinal cord response in rats after implants of polypyrrole and polyethylene glycol obtained by plasma. Journal of Materials Science: Materials in Medicine 19: 817-826. DOI 10.1007/s10856-007-3080-z.

Qi H, Ye Z, Ren H, Chen N, Zeng Q et al. (2016). Bioactivity assessment of PLLA/PCL/HAP electrospun nanofibrous scaffolds for bone tissue engineering. Life Sciences 148: 139-144.

Quinlan E, López-Noriega A, Thompson E, Kelly HM, Cryan SA et al. (2015). Development of collagen-hydroxyapatite scaffolds incorporating PLGA and alginate microparticles for the controlled delivery of rhBMP-2 for bone tissue engineering. Journal of Controlled Release 198: 71-79. DOI 10.1016/j.jconrel.2014.11.021.

Samadikuchaksaraei A, Gholipourmalekabadi M, Erfani Ezadyar E, Azami M, Mozafari M et al. (2016). Fabrication and in vivo evaluation of an osteoblast-conditioned nano-hydroxyapatite/ gelatin composite scaffold for bone tissue regeneration. Journal of Biomedical Materials Research Part A 104: 2001-2010. DOI 10.1002/jbm.a.35731.

Sha L, Chen ZF, Chen Z, Zhang AL (2016). Polylactic acid based nanocomposites: Promising safe and biodegradable materials in biomedical field. International Journal of Polymer Science Hierarchical Structured Polymeric Materials in Nanotechnology 2016: 1-11. DOI 10.1155/ 2016/6869154.

Tajbakhsh S, Hajiali FA (2017). A comprehensive study on the fabrication and properties of biocomposites of poly(lactic acid)/ceramics for bone tissue engineering. Materials Science and Engineering: C 70: 897-912. DOI 10.1016/j. msec.2016.09.008.

Venkatesan J, Anil S, Kim SK, Shim MS (2017). Chitosan as a vehicle for growth factor delivery: Various preparations and their applications in bone tissue regeneration. International Journal of Biological Macromolecules 104: 1383-1397. DOI 10.1016/j.ijbiomac.2017.01.072.

Yousefi AM, James PF, Akbarzadeh R, Subramanian A, Flavin C et al. (2016). Prospect of stem cells in bone tissue engineering: A review. Stem Cells International 2016: 1-13. DOI 10.1155/ 2016/6180487.

Zhang S, Ma B, Han J, Liu F, Wang S et al. (2018). Hydroxyapatite nanobelt/polylactic acid Janus membrane with osteoinduction/ barrier dual functions for precise bone defect repair. Acta Biomaterialia 71: 108-117. DOI 10.1016/j.actbio.2018.02.033. 